\title{
RNAi-mediated IARS2 knockdown inhibits proliferation and promotes apoptosis in human melanoma A375 cells
}

\author{
DONGMEI MA ${ }^{1}$, SONG LI ${ }^{1}$, XIAOJUAN NIE ${ }^{1}$, LAMEI CHEN $^{1}$, NAN CHEN $^{1}$, \\ DONGSHENG HOU ${ }^{2}$, XIUHONG $\mathrm{LIU}^{3}$ and BINBIN GAO ${ }^{4}$
}

\author{
Departments of ${ }^{1}$ Dermatology and ${ }^{2}$ Pathology, Shandong Provincial Hospital Affiliated to Shandong University, \\ Jinan, Shandong 250021; ${ }^{3}$ Department of Dermatology, The Sixth Affiliated Hospital of \\ Kun Ming Medical University, Yuxi, Yunnan 653100; ${ }^{4}$ Department of Oncology, \\ Shandong Provincial Hospital Affiliated to Shandong University, \\ Jinan, Shandong 250021, P.R. China
}

Received March 13, 2019; Accepted February 13, 2020

DOI: $10.3892 / \mathrm{ol} .2020 .11688$

\begin{abstract}
IARS2, which encodes the mitochondrial form of isoleucyl-tRNA synthetase, has been found to play an important role in a range of diseases, including cancer. However, the relationship between IARS2 and melanoma is still unclear. To evaluate the role of IARS2 in melanoma, we constructed a stable A375 cell line with IARS2 knockdown via lentivirus-mediated small interfering RNAs. The expression of IARS2 was measured by real time-quantitative Polymerase Chain Reaction and western blot analysis. Cell counting, 3-(4,5-dimethylthiazol-2-yl)-2,5-diphenyltetrazolium bromide assay, and colony formation assay were conducted to assess the effect of IARS2 on melanoma cell proliferation. Flow cytometry assay was used to determine cell apoptosis and cell cycle distribution in melanoma A375 cells. Finally, immunohistochemistry was employed to validate the expression of IARS2 protein in melanoma tissues. In this study it was found that IARS2 was highly expressed in melanoma cell lines. Furthermore, IARS2 protein also exhibited elevated expression in the tumour tissues obtained from melanoma patients. After suppression of the mRNA expression of IARS2, the proliferation and colony formation ability of the A375 cells were significantly inhibited, while the proportion of apoptotic A375 cells increased significantly, as indicated by an enhanced phosphatidylserine externalization and caspase 3/7 activity after IARS2 knockdown. Further investigations found that knockdown of IARS2 arrested cells in the G1 phase. The results suggested that IARS2 is critical for proliferation and apoptosis of melanoma cells.
\end{abstract}

Correspondence to: Dr Binbin Gao, Department of Oncology, Shandong Provincial Hospital Affiliated to Shandong University, 324 Jingwu Road, Jinan, Shandong 250021, P.R. China

E-mail: madmhospital@163.com

Key words: melanoma, growth, apoptosis, IARS2, RNAi-mediated knockdown

\section{Introduction}

Melanoma is the most aggressive type of skin cancer, the incidence of which has increased in recent decades (1-3). Despite the improvement in diagnosis and clinical therapy (4-8), there is still a high mortality rate among melanoma patients (9-11). In addition, melanoma cells develop drug resistance to clinical treatments and survival (12-14). Hence, there is an urgent need to identify novel drugs and strategies to improve melanoma treatment (15-18).

The function of aminoacyl-tRNA synthetases is to catalyse the aminoacylation of tRNA through their cognate amino acids (19). There are two forms of isoleucine-tRNA synthetase: Cytoplasmic and mitochondrial. IARS2 encodes for mitochondrial form of isoleucyl-tRNA synthetase (20). Recent studies have shown that IARS2 is involved in several diseases $(21,22)$. IARS2 expression is higher in tumour tissues than surrounding tissue and knockdown of IARS2 suppresses proliferation of the RKO cells (23). IARS2 mutation was found in a patient with neurotrophic keratitis and corneal opacification (21). Approximately 59\% of the colorectal cancers patients harbour a mutation at 5' upstream region of the mitochondrial IARS2 (24). Thus, IARS2 may be considered as a cancer-promoting gene (23,25-27).

To date, the association between IARS2 and melanoma remains unclear. In the present study, the function of IARS2 was elucidated in melanoma cell proliferation and apoptosis.

\section{Materials and methods}

Cell lines. The human melanoma cell lines A375, MUM-2B, and C918 were purchased from Cell Bank of the Chinese Academy of Sciences (Shanghai, China). A375 and C918 cell lines were cultured in Dulbecco's Modified Eagle Medium (GE Healthcare Life Sciences), while MUM-2B cell line was cultured in Roswell Park Memorial Institute (RPMI)-1640 medium (GE Healthcare Life Sciences) at $37^{\circ} \mathrm{C}$ in a $5 \% \mathrm{CO}_{2}$ incubator. Both media were supplemented with $10 \%$ fetal bovine serum (Thermo Fisher Scientific, Inc.), $100 \mathrm{U} / \mathrm{ml}$ penicillin, and $0.1 \mathrm{mg} / \mathrm{ml}$ streptomycin (Merck KGaA). 
$R T-q P C R$. Total RNA was extracted from A375, MUM-2B and C918 cells using TRIzol reagent (Thermo Fisher Scientific, Inc.) and was quantified using NanoDrop 2000 (Thermo Fisher Scientific, Inc.). A total of $2 \mu \mathrm{g}$ of RNA was reverse-transcribed to cDNA using miRNA 1st strand cDNA synthesis kit (Thermo Fisher Scientific, Inc.) according to the manufacturer's instruction. A total of $1 \mu 1$ of cDNA was assessed by SYBR Green real time-quantitative PCR (RT-qPCR). Primers were designed and synthesised by Guangzhou RiboBio Co., Ltd. The sequences are as follows: IARS2 forward, 5'-GGCAACCCATGACAATCCCA-3', and reverse, 5'-TGGACCTCCTTATGCAAACGG-3'; Glyceraldehyde-3-phosphate dehydrogenase (GAPDH) forward, 5'-TGA CTTCAACAGCGACACCCA-3' and reverse, 5'-CACCCT GTTGCTGTAGCCAAA-3'. RT-qPCR was performed at $95^{\circ} \mathrm{C}$ for $4 \mathrm{~min}$, then 40 cycles of $95^{\circ} \mathrm{C}$ for $15 \mathrm{sec}$ and $60^{\circ} \mathrm{C}$ for $45 \mathrm{sec}$ by a LightCycler 480 (Roche Diagnostics). The results were analysed by a GeneChip Scanner 3000 (Thermo Fisher Scientific, Inc.). All reactions were performed in triplicate. GAPDH was used to normalise expression. Relative expression level of target genes was calculated using $2^{-\Delta \Delta C q}$ method (28).

Lentiviral packaging and cell infection. The shIARS2 lentivirus and vector control were constructed by GeneChem, Inc. IARS2 oligonucleotides were designed to target the complementary DNA sequence (ACTTGCAGTCATCCATTAA). The hairpin sequence of shIARS2 was CCGGGTACTTGC AGTCATCCATTAATTCAAGAGATTAATGGATGACTG CAAGTACTTTTTG. The shRNA was synthesized and inserted into the GV115 vector (GeneChem, Inc.) at AgeI and EcoRI restriction sites. Lentivirus package was performed as described (29). Then shIARS2-lentivirus or negative control (shCtrl) lentivirus was transfected into A375 cells using Lipofectamine 2000 (Thermo Fisher Scientific, Inc.) according to the manufacturer's instructions. After $72 \mathrm{~h}$ of infection, the cells were observed under a fluorescence microscope, and subsequently harvested to determine knockdown efficiency by RT-qPCR and western blot analysis.

Western blot analysis. After $72 \mathrm{~h}$ of lentivirus infection, the cells were washed with cold phosphate buffer saline (PBS) and lysed in radioimmunoprecipitation assay (RIPA) lysis buffer supplemented with protease and phosphatase inhibitor cocktails for $15 \mathrm{~min}$. Cell lysate was centrifuged at $12,000 \mathrm{x} \mathrm{g}$ for $10 \mathrm{~min}$ at $4^{\circ} \mathrm{C}$ and the supernatants were collected. Protein concentration was measured by bicinchoninic acid protein assay. Equal amounts of total protein samples were analysed on sodium dodecyl sulphate polyacrylamide gel electrophoresis and transferred onto polyvinylidene fluoride (PVDF) membrane (EMD Millipore). PVDF membrane was blocked with $5 \%$ non-fat dried milk for $1 \mathrm{~h}$, and then incubated with the appropriate primary antibodies overnight at $4^{\circ} \mathrm{C}$. HRP-coupled secondary antibody was added and protein bands were visualized by enhanced chemiluminescence reagents (EMD Millipore). The antibodies for IARS2 (cat. no. ab66012) was purchased from Abcam. The antibodies for GAPDH (cat. no. sc-32233) and the secondary antibodies (cat. no. sc-2004) were purchased from Santa Cruz Biotechnology, Inc.
Cell growth assay. After infection with either shCtrl or shIARS2 lentivirus, the infected A375 cells were seeded into 96-well plates $\left(1 \times 10^{3}\right.$ cells per well). The plates were incubated at $37^{\circ} \mathrm{C}$ in $5 \% \mathrm{CO}_{2}$ for 5 days. During this period, cell number was imaged and counted by Celigo ${ }^{\circledR}$ Image Cytometer (Nexcelom Bioscience) every day.

Moreover, cell viability was measured every day by 3-(4,5-dimethylthiazol-2-Yl)-2,5-diphenyltetrazolium bromide (MTT) reagent. Melanoma cells transfected with shIARS2 or shCtrl were cultured at $37^{\circ} \mathrm{C}$ with $5 \% \mathrm{CO}_{2}$ for $24 \mathrm{~h}$, then detached with $0.25 \%$ trypsin solution, and placed in 96-well plates at a final concentration of 2,000 cells/ml. MTT assay was performed at 24, 48, 72, 96 and $120 \mathrm{~h}$ following transfection. Briefly, the culture medium was replaced and $10 \mu \mathrm{l}$ MTT solution/well was added. The cells with MTT were incubated for $4 \mathrm{~h}$ at $37^{\circ} \mathrm{C}$ in $5 \% \mathrm{CO}_{2}$. Post incubation, the cells were washed and $200 \mu 1$ dimethyl sulfoxide/well was added. Finally, the cells were incubated at room temperature for $10 \mathrm{~min}$. Absorbance of stained supernatants was detected at $490 \mathrm{~nm}$ using a spectrophotometer (Thermo Fisher Scientific, Inc.). Cell viability / proliferation is directly proportional to the absorbance rate. The experiment was performed in triplicate.

Colony formation assay. A375 shCtrl and IARS2 knockdown cells were plated $(1,000$ cells/well) in a 6 -well culture plate in triplicate. The culture medium were refreshed every three days for 10 days or until colonies were formed. Following colony formation, they were washed twice with PBS, fixed with $4 \%$ paraformaldehyde for $30 \mathrm{~min}$, and stained with $0.4 \%$ crystal violet for $15 \mathrm{~min}$. The number of colonies containing $\geq 50$ cells was counted under a microscope.

Flow cytometry analysis. Cell apoptosis was evaluated by flow cytometry according to the manufacturer's instructions. After A375 cells infected with shIARS2-lentivirus or shCtrl lentivirus were cultured in 6-well plates for 5 days, they were collected and washed with cold PBS. Then, a $100 \mu \mathrm{l}$ cell suspension was prepared and stained with $5 \mu \mathrm{l}$ of Annexin V-APC and $5 \mu \mathrm{l}$ of 7-AAD (BD Biosciences) for $15 \mathrm{~min}$. The ratio of apoptotic cells was analysed by a flow cytometer (EMD Millipore).

For analysis of caspase $3 / 7$ activity, the lentivirus-infected A375 cells $\left(2 \times 10^{4}\right.$ cells per well) were cultured in 96-well plates for 5 days. Then $100 \mu$ l Caspase-Glo 3/7 reagent (Promega Corporation) was added and the cells were incubated for $2 \mathrm{~h}$. The luminescence of each sample was measured in a luminometer plate reader according to the manufacturer's instruction.

For cell cycle analysis, the lentiviral vector-transduced A375 cells were labelled with propidium iodide (Merck KGaA) and analysed using a flow cytometer. Annexin V-APC-positive cells were considered apoptotic regardless of the 7-AAD status.

Clinical samples and immunohistochemistry (IHC) staining. In this study, tumour samples of patients diagnosed with malignant melanoma between January 2012 and December 2017 were collected. A total of 30 melanoma tissues and 30 surrounding skin tissue were examined for the expression of IARS2 protein using IHC. All paraffin-embedded tissues were fixed in $4 \%$ paraformaldehyde. Briefly, the slides were deparaffinized with 
A

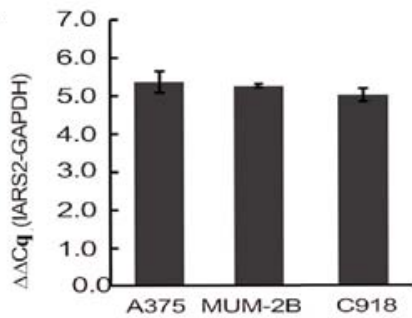

C
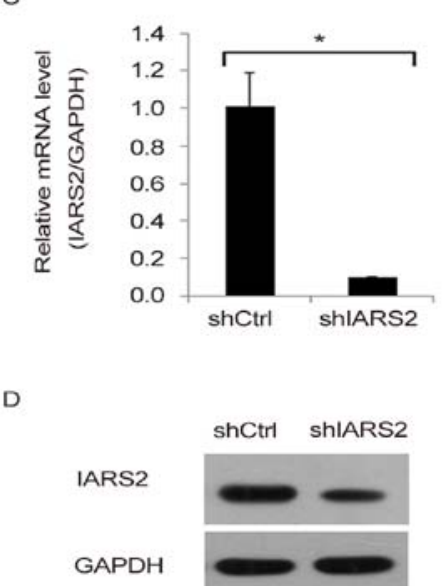

B

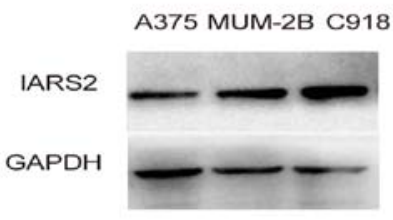

$E$
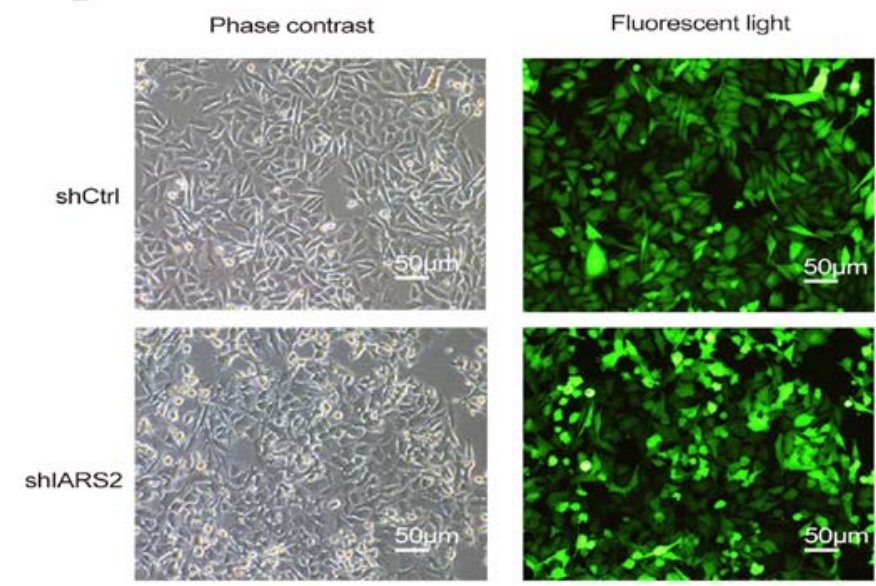

Figure 1. Knockdown of IARS2 expression in A375 cells. (A) IARS2 mRNA expression levels in three melanoma cancer cell lines. IARS2 mRNA expression was measured in the three melanoma cell lines, A375, MUM-2B, and C918, using quantitative RT-PCR assay. GAPDH mRNA expression levels were used to normalize expression levels of IARS2. Data from multiple groups were analyzed by using one-way ANOVA, with the Tukey's post hoc test. P $>0.05$. (B) IARS2 protein levels in three melanoma cancer cell lines. (C) Assessment of efficiency of infection of A375 cells with shIARS2 or shCtrl lentiviral vectors. IARS2 mRNA expression in A375 cells was determined by quantitative RT-PCR after infection with shIARS2 lentivirus or shCtrl lentivirus. T-test was used for comparison between two groups. "P<0.05. (D) IARS2 protein expression in A375 cells was determined by western blot analysis in shIARS2 A375 cells compared with shCtrl A375 cells. (E) A375 cells were infected with IARS2-siRNA or NC lentivirus and examined by fluorescence microscopy and light microscopy at day 3 post-infection. Note that most of the cells express GFP. Magnification, x100. Representative images of the cultures are shown.

xylene and rehydrated in graded alcohol. Antigen was retrieved and endogenous peroxidase activity was blocked by $3 \%$ hydrogen peroxide for $30 \mathrm{~min}$. The slides were then incubated with goat serum to block the non-specific proteins (Bioz, Inc.). Subsequently, the slides were incubated overnight at $4^{\circ} \mathrm{C}$ with primary antibody against IARS2 (Abcam), and finally incubated with biotinylated secondary antibody (Bioz, Inc.) for $1 \mathrm{~h}$ at room temperature. Tris buffered saline with Tween-20 was used in the washing steps. All sections were scored blindly by two investigators under a light microscope and recorded. The tissue staining was scored by the percentage of positive staining cells. The percentage of positive cells was scored as negative (score $0:<1 \%$ of tumour cells stain positive, or 1 : $1-5 \%$ of tumour cells stain positive) or positive (score 2: 6-25\%, score 3: $26-50 \%$, score $4:>50 \%$ positive of the tumour area). Scores of each subgroup of clinicopathological parameters are presented as mean \pm standard deviation (SD). Man-Whitney U test was applied for comparisons between two groups.

Ethical approval was obtained from the clinical research Ethics Committee of Shandong Provincial Hospital affiliated to Shandong University (2016142) (Jinan, China). Written informed consent for the acquisition and use of tissue samples was obtained from all patients.

Statistical analysis. Statistical analysis were performed using SPSS 16.0 (SPSS, Inc.). The statistical data for each group are presented as the mean \pm SD. T-test was used for comparison between two groups. Continuous data from multiple groups were analyzed by using one-way ANOVA, with the Tukey's post hoc test. $\mathrm{P}<0.05$ was accepted as statistically significant.

\section{Results}

Expression of IARS2 in four melanoma cell lines. To explore the role of IARS2 in human melanoma development, we first detected the expression of IARS 2 in three human melanoma cell lines, including A375, MUM-2B, and C918, using RT-qPCR. We also detected the protein levels of IARS2 in the three melanoma cell lines. The results showed that IARS2 mRNA and protein were expressed in all three cell lines (Fig. 1A and B).

Knockdown of IARS2 in A375 cells. To further investigate the function of IARS2 in human melanoma, we conducted lentivirus-mediated knockdown of IARS2 in the human melanoma cell line A375, and quantified its expression by RT-qPCR (Fig. 1C-E). By day 3 post-infection, fluorescence microscopy was used to detect the GFP expression. As shown in Fig. 1E, the proportion of infected cells was $>80 \%$. As shown in Fig. 1C, IARS2 mRNA was significantly downregulated in A375 cells infected with shIARS2 lentivirus compared to cells treated with shCtrl lentivirus $(\mathrm{P}<0.05)$. Knockdown efficiency was also examined by western blot analysis, IARS2 

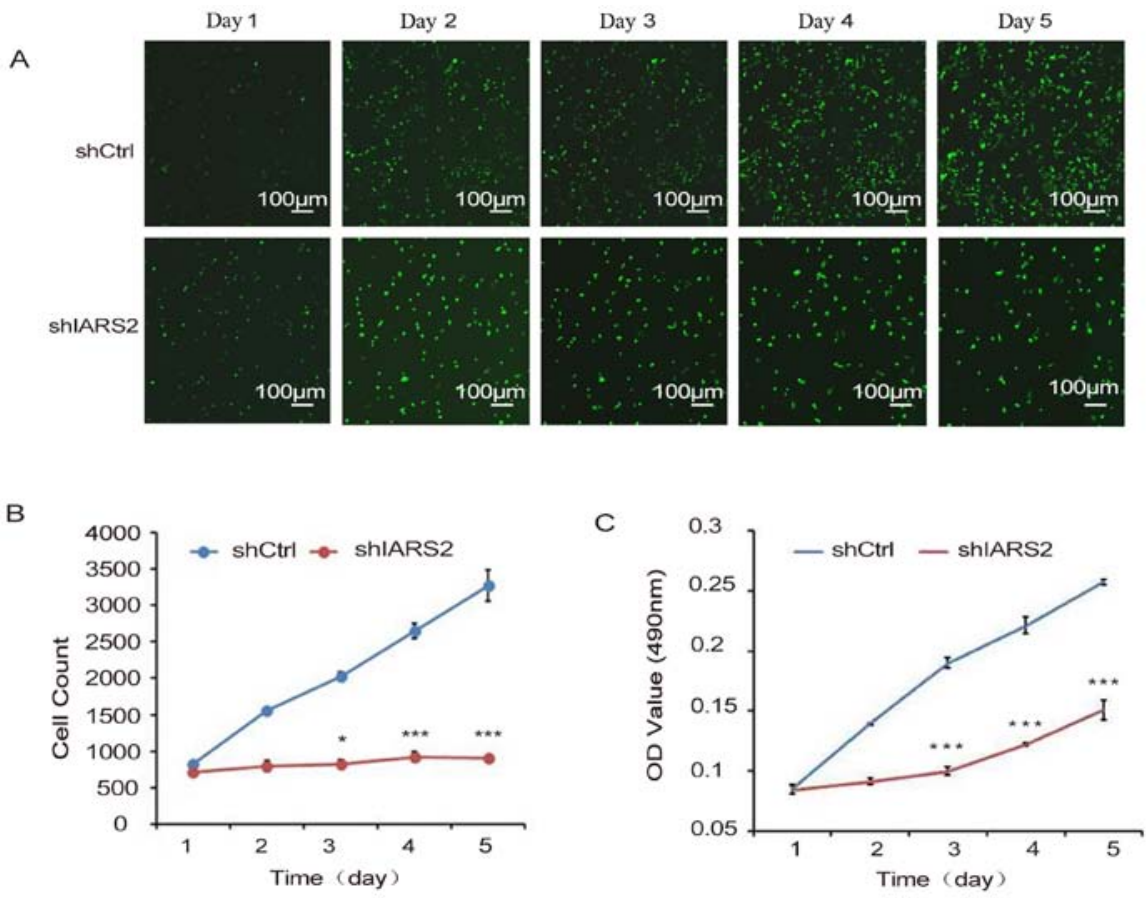

Figure 2. Effect of IARS2 knockdown on A375 cell proliferation. (A) A375 cell infection with shIARS2 lentivirus or shCtrl lentivirus was imaged and analysed using a Celigo cytometer. (B) The number of cells was counted every day for 5 days (shCtrl vs. shIARS2). (C) Proliferation of shIARS2 A375 cells and shCtrl A375 cells was evaluated by MTT assay. Bars represent the mean \pm standard deviation. T-test was used for comparison between shCtrl and shIARS2 groups at each time point. ${ }^{*} \mathrm{P}<0.05,{ }^{* * * *} \mathrm{P}<0.001$. Data from multiple time points of either shCtrl or shIARS2 group were analyzed by using one-way ANOVA, with the Tukey's post hoc test. Compared to day $1, \mathrm{P}<0.05$; compared to day $2, \mathrm{P}<0.05$; compared to day $3, \mathrm{P}<0.05$; compared to day $4, \mathrm{P}<0.05$.
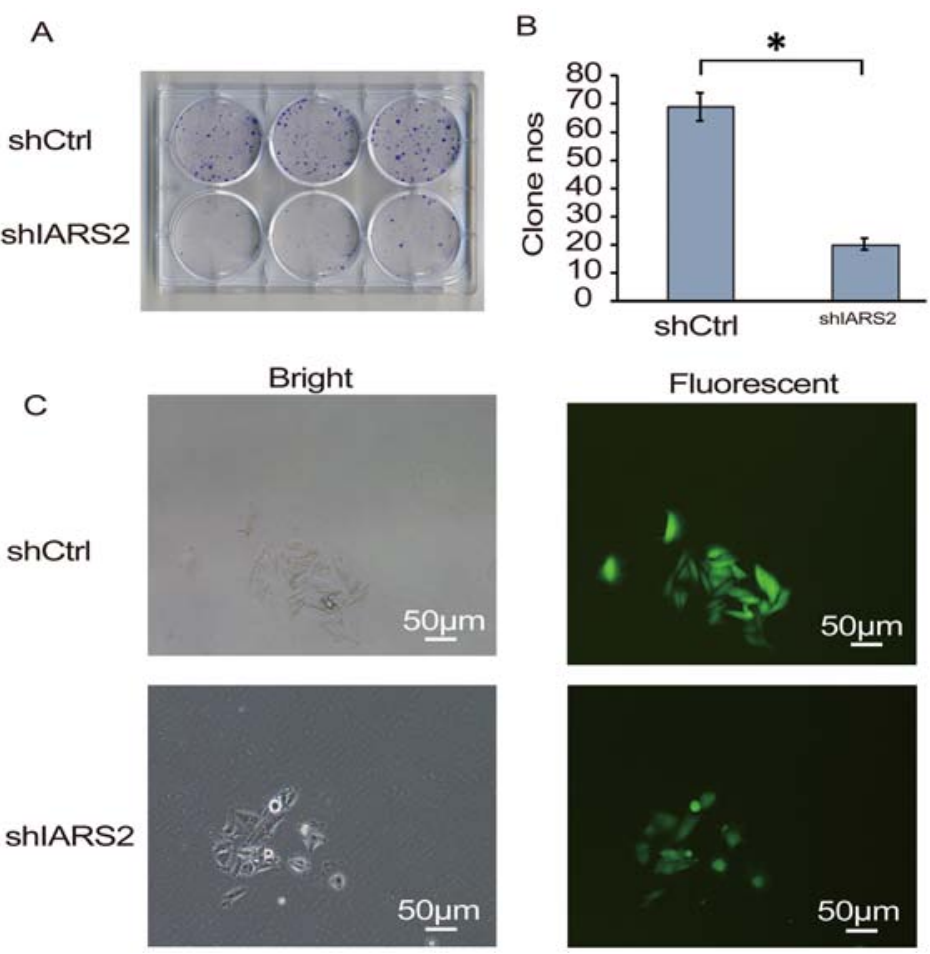

Figure 3. Effect of IARS2 knockdown on colony formation ability of A375 cells. (A) A375 cells were seeded in 6-well plates (1,000 cells/well) for colony formation assay. Plate clone formation assay showed that suppression of IARS2 expression significantly inhibited cell proliferation compared to shCtrl cells. (B) T-test was used for comparison between shCtrl and shIARS2 groups at each time point. "P<0.05. (C) Clone formation of cells in shIARS2 and shCtrl groups (magnification, x100).

protein expression was significantly reduced in shIARS2 A375 cells compared to shCtrl A375 cells (Fig. 1D). All the results demonstrated effective knockdown of IARS2 in A375 cells. 
A

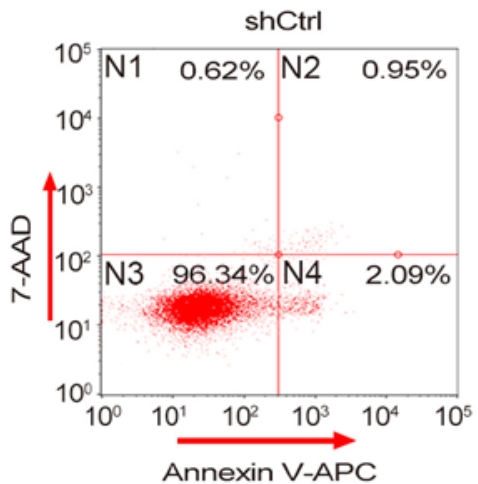

ShIARS2

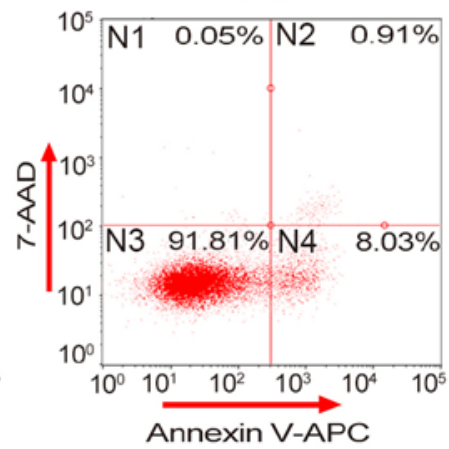

$\mathrm{B}$

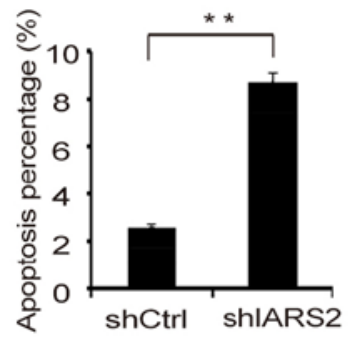

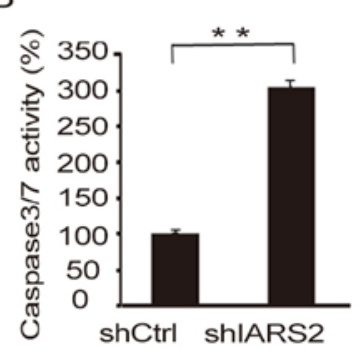

C
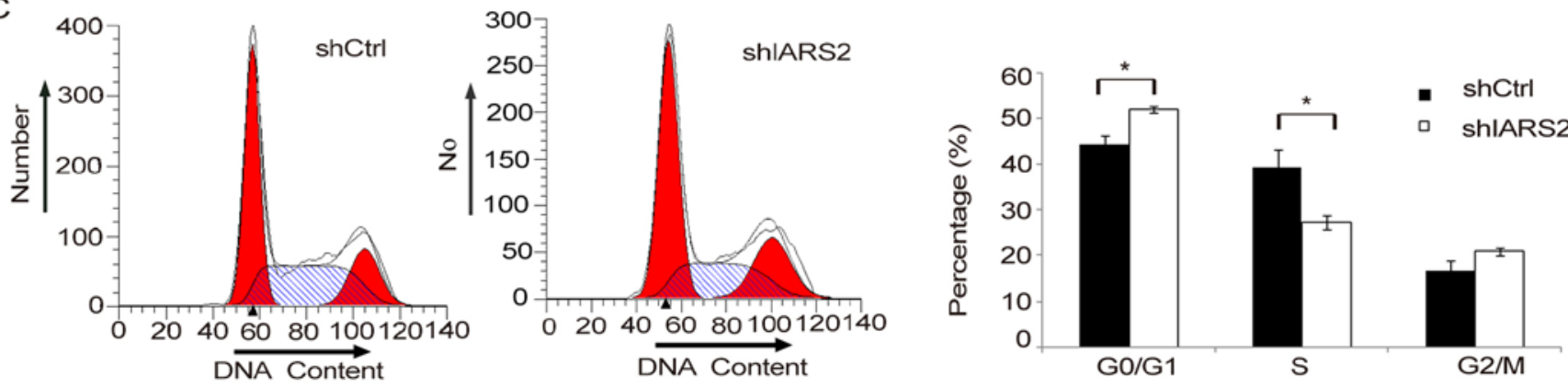

Figure 4. Effect of IARS2 knockdown on A375 cell apoptosis and cell cycle. (A) Cell apoptosis was determined using Annexin V-APC/7-AAD staining and flow cytometry. Analysis of data from quadrant regions in the dot plots were interpreted as follows: N1, the top left quadrant designates necrotic cells that are 7-AAD-positive and annexin V-APC-negative; N2, the top right quadrant includes late apoptotic cells that are annexin V-APC and 7-AAD-positive; N3, the bottom left quadrant specifies unstained viable cells that are annexin V-APC and 7-AAD double-negative; and N4, the right bottom quadrant denotes early apoptotic cells that are annexin V-APC-positive, but 7-AAD-negative. The number of apoptotic shIARS2 A375 cells was significantly higher than the number of apoptotic shCtrl A375 cells. (B) Caspase 3/7 activity was significantly higher in shIARS2 A375 cells compared to shCtrl A375 cells. T-test was used for comparison between shCtrl and shIARS2 groups. ${ }^{* *} \mathrm{P}<0.01$. (C) Cell cycle was assessed in A375 cells after transfection with the indicated shRNAs. Representative flow-cytograms are shown. Bar graphs indicate the mean percentages. T-test was used for comparison between shCtrl and shIARS2 groups at each time point. ${ }^{*} \mathrm{P}<0.05$.

Knockdown of IARS2 inhibits human melanoma cell growth and colony formation. To determine the effect of IARS2 expression on human melanoma cell growth, we infected A375 cells with shIARS2 lentivirus or shCtrl lentivirus, and analysed the cell growth every day for 5 days by counting the number of cells. We found that the cell growth rate of shIARS2 A375 cells was dramatically decreased compared to shCtrl A375 cells (Fig. 2A-B).

To confirm the inhibitory effect of IARS2 on human melanoma cell growth, we detected the proliferation of A375 cells by MTT assay every day for 5 days. MTT assay showed that melanoma cell proliferation was significantly inhibited in shIARS2 A375 cells compared with shCtrl A375 cells (Fig. 2C).

Furthermore, a colony formation assay was performed. The results of the assay confirmed that IARS2 knockdown significantly inhibited the colony formation of A375 cells (Fig. 3; $\mathrm{P}<0.05$ ).

Effect of IARS2 knockdown on apoptosis and cell cycle. As is well known, apoptosis is associated with the mechanism of tumour progression. We detected whether knockdown of IARS2 affects cell apoptosis. Double staining with Annexin $\mathrm{V}-\mathrm{APC}$ and 7-AAD showed that the proportion of apoptotic A 375 cells in the shCtrl and shIARS2 groups was $2.62 \pm 0.35 \%$ and $8.77 \pm 1.32 \%$, respectively $(\mathrm{P}<0.05)$, suggesting that knockdown of IARS2 significantly enhanced cell apoptosis compared with the negative control group (Fig. 4A). Moreover, this result was further validated by caspase $3 / 7$ activity analysis. We observed that caspase 3 and 7 activities were significantly upregulated in shIARS2 A375 cells compared to shCtrl A375 cells (Fig. 4B). Taken together, these data suggested that knockdown of IARS2 promoted human melanoma cell apoptosis.

Cell cycle analysis showed that knockdown of IARS2 affected the distribution of cell cycle phases (Fig. 4C). Compared to control cells, the proportion of G0/G1 cells increased, while that of $\mathrm{S}$ cells decreased in IARS2-siRNA group (Fig. 4C, $\mathrm{P}<0.05$ ).

Expression of IARS2 protein in melanoma tissues. To further demonstrate the role of IARS2 in human melanoma development, we detected the expression of IARS2 in clinical melanoma patient tissues by IHC (Fig. 5). Tumour tissues and surrounding skin tissues from 30 patients were examined for the expression of IARS 2 protein in this study. The male-tofemale ratio was 1.3:1. The age of the patients ranged from 23-79 years, and a median age was 61 years. The results showed that expression of IARS2 was higher in tumour tissues (Fig. 5A) than in adjacent skin tissue (Fig. 5B and $\mathrm{C} ; \mathrm{P}<0.05$ ). The staining of the IARS2 revealed that it was mainly localised in the cytoplasm with brown positive granules in tumour 
A

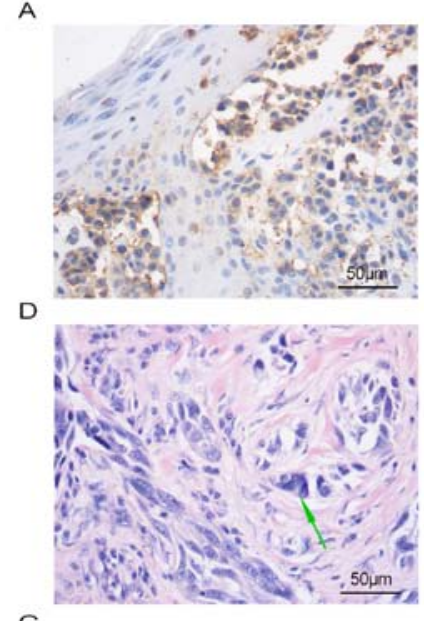

G

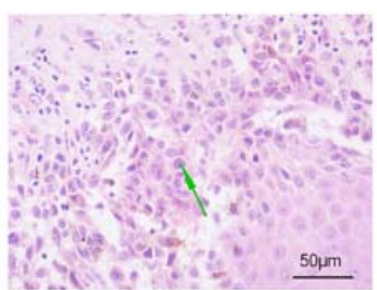

B

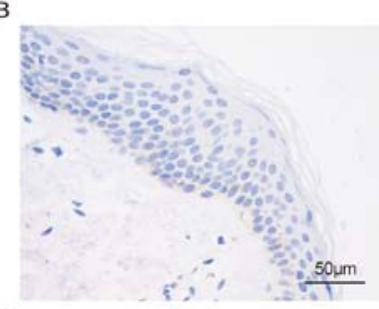

E

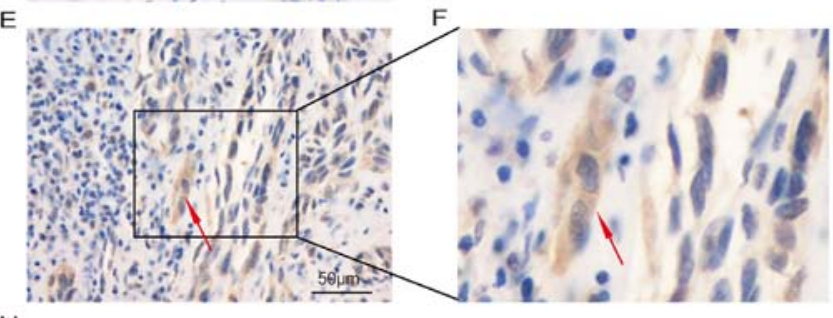

$\mathrm{H}$

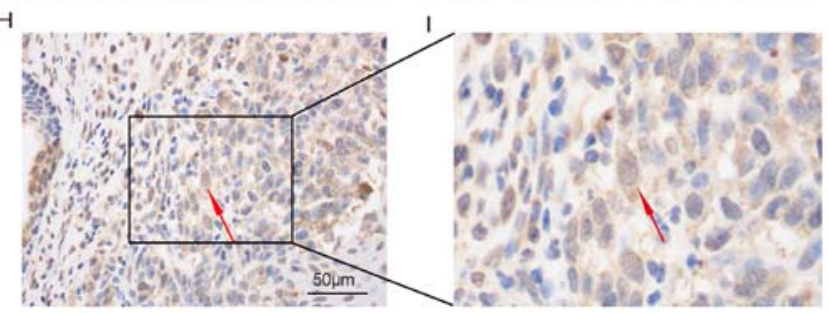

Figure 5. Expression of IARS2 protein in melanoma tissues. (A) Representative micrographs of the staining patterns of IARS2 in melanoma. (B) The staining of IARS2 in normal skin tissue was negative. (C) The expression of IARS2 was higher in the melanoma tumour cells than in the normal skin tissue. T-test was used for comparison between tumour tissues and normal skin tissues. ${ }^{* *} \mathrm{P}<0.01$. (D-E): HE staining (green arrow indicates melanoma cells) and (D) IHC staining of IARS2, (E) in a tissue section obtained from an IARS2-positive melanoma patient. (F) A partially enlarged image of Fig. 5E. (G-H) HE staining (green arrow indicates melanoma cells) and (G) IHC staining of IARS2, (H) in a tissue section obtained from another IARS2-positive melanoma patient. (I) A partially enlarged image of Fig. 5E. IHC staining in tissue sections of melanoma lesions (microphotograph obtained with a magnification, $\mathrm{x} 400$; scale bar: $50 \mu \mathrm{m}$ ). Positive cells stained brown. Staining was primarily observed in the cell cytoplasm (red arrows).

tissues (Fig. 5A, E and H). Fig. 5E shows the IHC staining of IARS2 in tumour tissue obtained from an IARS2-positive melanoma patient (pathological no. S1302026). Fig. 5H shows the same staining pattern of IARS 2 in a tissue section obtained from another IARS2-positive melanoma patient (pathological no. S1408271).

\section{Discussion}

Melanoma is the most dangerous skin cancer and accounts for the most skin cancer-related deaths worldwide $(30,31)$. The improvement in diagnosis and clinical therapy has led to 5-year survival of melanoma patients. However, doctors have been able to do very little to treat metastatic melanoma. In addition, development and progression of melanoma still remain poorly understood. In order to identify more effective therapeutic strategy for treating melanoma, it is very important to identify the novel factors that are related to melanoma incidence and progression and to unravel the underlying mechanisms $(32,33)$. Hence, there is an urgent need to identify novel factors associated with melanoma and strategies to improve melanoma treatment.

Aminoacyl-tRNA synthetases (AARS) identify the cognate amino acid to catalyse the aminoacylation of tRNA (34). Apart from protein synthesis, recent studies have shown that AARS are also involved in several other pathophysiological processes, including inflammation, angiogenesis, and tumorigenesis. It has been reported that genetic mutation in the glycyl-tRNA synthetase coding region is associated with Charcot-Marie-Tooth disease type 2D and distal spinal muscular atrophy type V (35). Human tyrosyl-tRNA synthetase promotes angiogenesis but human tryptophanyltRNA synthetase suppresses vascular growth (36). The human glutamyl-tRNA synthetase regulates cell apoptosis via interaction with apoptosis signal-regulating kinase 1 (37). AARS are aberrantly expressed in several types of cancers, suggesting their potential association with tumorigenesis (38).

IARS2 encodes for mitochondrial form of isoleucyl-tRNA synthetase, which belongs to the class-I AARS family. The mitochondrial isoleucine-tRNA synthetase was first synthesized in the cytoplasm, and then, transported into the mitochondrion. Recent studies have shown that IARS2 is involved in several diseases. In 2014, the clinical features of patients with IARS2 mutation were reported including growth hormone deficiency, partial deafness, cataracts, and peripheral neuropathy (22). High expression level of IARS2 attenuated the clinical manifestations of patients with mtDNA mutation (39). Importantly, IARS2 exhibited a vital role in the incidence and development of colon cancer (23). IARS2 was highly expressed in human colon cancer tissue compared to surrounding tissues. Several studies have indicated that IARS2 may be a cancer-promoting gene $(23,25-28)$.

In our study, melanoma cancer cell lines, A375, MUM-2B, and C918, were selected for the experiment. First it was found that the mRNA expression level of IARS2 was high in all four cell lines (Fig. 1A). IHC analysis also confirmed a higher expression of IARS2 protein in clinical melanoma tissues than in surrounding tissues (Fig. 5). Further cytological 
study showed that knockdown of IARS2 inhibited A375 cell proliferation and colony formation (Figs. 2 and 3). Flow cytometry revealed that knockdown of IARS2 promoted cell apoptosis and influenced cell cycle distribution (Fig. 4). Our results suggested that IARS2 may be involved in the development and progression of melanoma.

MicroRNAs (miRNAs) are a class of small non-coding RNAs that regulate gene expression at the post-transcriptional level. They play pivotal roles during several biological processes, including tumorigenesis in melanoma $(40,41)$. MiRNA expression levels are regulated in response to various factors, including toxicant stresses in the environment (42). Based on their genomic location, miRNAs are generally classified as 'intergenic' or 'intronic.' The 'intronic' miRNAs share the promoter with their host gene and they are usually expressed along with their host gene indicating the functional association with their host gene (43). IARS2 is the host gene of miR-215, a member of the miR-192/215 family. In colon tissues, miR-215 expression was shown to be positively correlated with expression of its host gene, IARS2, which indicated that miR215 was transcribed together with IARS2 (44). The expression of miR-215 might be abnormal after IARS2 was knocked down in A375 melanoma cells. Previous studies showed that miR-215 plays an important role in tumour development and metastasis (45-47). It may promote cell proliferation, repress apoptosis, and alter the cell cycle $(45,46)$. Thus, the inhibition of proliferation of A375 cells with IARS2 knockdown might be also associated with the alteration of miR-215 expression in shIARS2 cells. However, this speculation still needs to be proved.

In conclusion, our study showed that downregulation of IARS 2 expression by lentivirus-delivered small interfering RNA in A375 cells inhibited cell proliferation and colony formation, induced cell cycle arrest, and upregulated caspase 3/7 activity, which then promoted cell death through apoptosis. Therefore, knockdown of IARS 2 might be a potential therapeutic approach for melanoma in which IARS2 is highly expressed.

\section{Acknowledgments}

We thank Dr Lei Zhao (Institute of Basic Medical Sciences, Qilu Hospital of Shandong University (Jinan, China) for providing technical assistance and experimental materials.

\section{Funding}

This project was sponsored by a grant from the Key Research and Development Program of Shandong Province (grant nso. 2015GSF118171 and 2016GSF201212).

\section{Availability of data and materials}

The datasets used and/or analysed during the present study are available from the corresponding author on reasonable request.

\section{Authors' contributions}

DM conceived the study and drafted the manuscript. DM was responsible for RNA extraction and quantitative RT-PCR assay. XN and LC performed the western blot assay and cell growth assay. SL was responsible for lentiviral packaging and cell infection. NC and DH conducted colony formation assay and cell apoptosis assay. XL and BG were responsible for clinical samples and IHC staining. All authors read and approved the final manuscript.

\section{Ethics approval and consent to participate}

Ethical approval was obtained from the clinical research Ethics Committee of Shandong Provincial Hospital affiliated to Shandong University (approval no. 2016142; Jinan, China). Written informed consent for the acquisition and use of tissue samples was obtained from all patients.

\section{Patient consent for publication}

Not applicable.

\section{Competing interests}

The authors declare that they have no competing interests.

\section{References}

1. Rastrelli M, Tropea S, Rossi CR and Alaibac M: Melanoma: epidemiology, risk factors, pathogenesis, diagnosis and classification. In Vivo 28: 1005-1011, 2014.

2. Minini R, Rohrmann S, Braun R, Korol D and Dehler S: Incidence trends and clinical-pathological characteristics of invasive cutaneous melanoma from 1980 to 2010 in the Canton of Zurich, Switzerland. Melanoma Res 27: 145-151, 2017.

3. Lin AY, Wang PF, Li H and Kolker JA: Multicohort model for prevalence estimation of advanced malignant melanoma in the USA: An increasing public health concern. Melanoma Res 22: 454-459, 2012.

4. Read T, Webber S, Thomas J, Wagels M, Schaider H, Soyer HP and Smithers BM: Protocol for the TIDAL melanoma study: Topical imiquimod or diphenylcyclopropenone for the management of cutaneous in-transit melanoma metastases-a phase II, single centre, randomised, pilot study. BMJ Open 7: e016816, 2017.

5. Pike E, Hamidi V, Saeterdal I, Odgaard-Jensen J and Klemp M: Multiple treatment comparison of seven new drugs for patients with advanced malignant melanoma: A systematic review and health economic decision model in a Norwegian setting. BMJ Open 7: e014880, 2017.

6. Shen J, Lei QQ, Chen X, Cao C and Cen Y: Diagnostic performance of micropthalmia transcription factor for melanoma: A systematic review and meta-analysis. Eur Rev Med Pharmacol Sci 18: 798-805, 2014

7. Sun J, Zager JS and Eroglu Z: Encorafenib/binimetinib for the treatment of BRAF-mutant advanced, unresectable, or metastatic melanoma: Design, development, and potential place in therapy. OncoTargets Ther 11: 9081-9089, 2018.

8. Cosgarea I, Ritter C, Becker JC, Schadendorf D and Ugurel S: Update on the clinical use of kinase inhibitors in melanoma. J Dtsch Dermatol Ges 15: 887-893, 2017.

9. Robsahm TE, Helsing P, Nilssen Y, Vos L, Rizvi SMH, Akslen LA and Veierød MB: High mortality due to cutaneous melanoma in Norway: A study of prognostic factors in a nationwide cancer registry. Clin Epidemiol 10: 537-548, 2018.

10. Boniol M, Autier P and Gandini S: Melanoma mortality following skin cancer screening in Germany. BMJ Open 5: e008158, 2015.

11. Bottoni U,Paolino G, Didona D, Corsetti P, Clerico R, Cantisani C, Richetta AG, Arcidiacono V, Scali E, Pranteda G: Improvement of survival in patients with melanoma and non-melanoma skin cancers compared to patients without double cutaneous malignancies. Eur Rev Med Pharmacol Sci 19: 1640-1644, 2015.

12. Filitis DC, Rauh J and Mahalingam M: The HGF-cMET signaling pathway in conferring stromal-induced BRAF-inhibitor resistance in melanoma. Melanoma Res 25: 470-478, 2015. 
13. Mak G, Arkenau HT and Chin M: Resistance surveillance in a BRAF mutant melanoma patient on long-term BRAF-inhibitor treatment. Melanoma Res 24: 408-412, 2014

14. Cohen-Solal KA, Kaufman HL and Lasfar A: Transcription factors as critical players in melanoma invasiveness, drug resistance, and opportunities for therapeutic drug development. Pigment Cell Melanoma Res 31: 241-252, 2018.

15. Gladfelter P, Darwish NHE and Mousa SA: Current status and future direction in the management of malignant melanoma. Melanoma Res 27: 403-410, 2017.

16. Najem A, Krayem M, Perdrix A, Kerger J, Awada A, Journe F and Ghanem G: New drug combination strategies in melanoma: Current status and future directions. Anticancer Res 37: 5941-5953, 2017.

17. Zhu Z, Liu W and Gotlieb V: The rapidly evolving therapies for advanced melanoma - Towards immunotherapy, molecular targeted therapy, and beyond. Crit Rev Oncol Hematol 99: 91-99, 2016.

18. Bogusławska $\mathbf{J}$ and Małecki M: siRNA preparations in gene therapy of melanoma. Med Wieku Rozwoj 17: 196-201, 2013

19. Rajendran V, Kalita P, Shukla H, Kumar A and Tripathi T: Aminoacyl-tRNA synthetases: Structure, function, and drug discovery. Int J Biol Macromol 111: 400-414, 2018.

20. LeeSW,ChoBH,ParkSGandKimS: Aminoacyl-tRNA synthetase complexes: Beyond translation. J Cell Sci 117: 3725-3734, 2004.

21. Jabbour S and Harissi-Dagher M: Recessive mutation in a nuclear-encoded mitochondrial tRNA synthetase associated with infantile cataract, congenital neurotrophic keratitis, and orbital myopathy. Cornea 35: 894-896, 2016.

22. Schwartzentruber J, Buhas D, Majewski J, Sasarman F, PapillonCavanagh S, Thiffault I, Sheldon KM, Massicotte C, Patry L, Simon M, et al; FORGE Canada Consortium: Mutation in the nuclear-encoded mitochondrial isoleucyl-tRNA synthetase IARS 2 in patients with cataracts, growth hormone deficiency with short stature, partial sensorineural deafness, and peripheral neuropathy or with Leigh syndrome. Hum Mutat 35: 1285-1289, 2014.

23. Zhong L, Zhang Y, Yang JY, Xiong LF, Shen T, Sa YL, O'Yang YM, Zhao SH and Chen JY: Expression of IARS2 gene in colon cancer and effect of its knockdown on biological behavior of RKO cells. Int J Clin Exp Pathol 8: 12151-12159, 2015.

24. Miyaki M, Iijima T, Shiba K, Aki T, Kita Y, Yasuno M, Mori T, Kuroki T and Iwama T: Alterations of repeated sequences in 5 upstream and coding regions in colorectal tumors from patients with hereditary nonpolyposis colorectal cancer and Turcot syndrome. Oncogene 20: 5215-5218, 2001.

25. Yin J, Liu W, Li R, Liu J, Zhang Y, Tang W and Wang K: IARS2 silencing induces non-small cell lung cancer cells proliferation inhibition, cell cycle arrest and promotes cell apoptosis. Neoplasma 63: 64-71, 2016

26. Fang Z, Wang X, Yan Q, Zhang S and Li Y: Knockdown of IARS2 suppressed growth of gastric cancer cells by regulating the phosphorylation of cell cycle-related proteins. Mol Cell Biochem 443: 93-100, 2018.

27. Li H, Tian Y, Li X, Wang B, Zhai D, Bai Y, Dong C and Chao X: Knockdown of IARS2 inhibited proliferation of acute myeloid leukemia cells by regulating p53/p21/PCNA/eIF4E pathway. Oncol Res 27: 673-680,2019.

28. Livak KJ and Schmittgen TD: Analysis of relative gene expression data using real-time quantitative PCR and the 2(-Delta Delta C(T)) method. Methods 25: 402-408, 2001.

29. Lois C, Hong EJ, Pease S, Brown EJ and Baltimore D: Germline transmission and tissue-specific expression of transgenes delivered by lentiviral vectors. Science 295: 868-872, 2002.

30. Garbe C, Peris K, Hauschild A, Saiag P, Middleton M, Bastholt L, Grob JJ, Malvehy J, Newton-Bishop J, Stratigos AJ, et al; European Dermatology Forum (EDF); European Association of Dermato-Oncology (EADO); European Organisation for Research and Treatment of Cancer (EORTC): Diagnosis and treatment of melanoma. European consensus-based interdisciplinary guideline - Update 2016. Eur J Cancer 63: 201-217, 2016.
31. Miller KD, Siegel RL, Lin CC, Mariotto AB, Kramer JL, Rowland JH, Stein KD, Alteri R and Jemal A: Cancer treatment and survivorship statistics, 2016. CA Cancer J Clin 66: 271-289, 2016.

32. Tas F: Metastatic behavior in melanoma: Timing, pattern, survival, and influencing factors. J Oncol 2012: 647684, 2012.

33. Sarkar D, Leung EY, Baguley BC, Finlay GJ and AskarianAmiri ME: Epigenetic regulation in human melanoma: Past and future. Epigenetics 10: 103-121, 2015.

34. Diodato D, Ghezzi D and Tiranti V: The mitochondrial aminoacyl tRNA synthetases: Genes and syndromes. Int J Cell Biol 2014: 787956, 2014.

35. Antonellis A, Ellsworth RE, Sambuughin N, Puls I, Abel A, Lee-Lin SQ, Jordanova A, Kremensky I, Christodoulou K, Middleton LT, et al: Glycyl tRNA synthetase mutations in Charcot-Marie-tooth disease type 2D and distal spinal muscular atrophy type V. Am J Hum Genet 72: 1293-1299, 2003.

36. Yao P and Fox PL: Aminoacyl-tRNA synthetases in medicine and disease. EMBO Mol Med 5: 332-343, 2013.

37. Ko YG, Kim EY, Kim T, Park H, Park HS, Choi EJ and Kim S: Glutamine-dependent antiapoptotic interaction of human glutaminyl-tRNA synthetase with apoptosis signal-regulating kinase 1. J Biol Chem 276: 6030-6036, 2001.

38. Kim S, You S and Hwang D: Aminoacyl-tRNA synthetases and tumorigenesis: More than housekeeping. Nat Rev Cancer 11: 708-718, 2011.

39. Perli E, Giordano C, Tuppen HA, Montopoli M, Montanari A, Orlandi M, Pisano A, Catanzaro D, Caparrotta L, Musumeci B, et al: Isoleucyl-tRNA synthetase levels modulate the penetrance of a homoplasmic m.4277T $>C$ mitochondrial tRNA(Ile) mutation causing hypertrophic cardiomyopathy. Hum Mol Genet 21: 85-100, 2012.

40. Fattore L, Costantini S, Malpicci D, Ruggiero CF, Ascierto PA, Croce CM, Mancini R and Ciliberto G: MicroRNAs in melanoma development and resistance to target therapy. Oncotarget 8: 22262-22278, 2017.

41. Bennett PE, Bemis L, Norris DA and Shellman YG: miR in melanoma development: miRNAs and acquired hallmarks of cancer in melanoma. Physiol Genomics 45: 1049-1059, 2013.

42. Gonzalez H, Lema C, Kirken RA, Maldonado RA, VarelaRamirez A and Aguilera RJ: Arsenic-exposed keratinocytes exhibit differential microRNAs expression profile; Potential implication of miR-21, miR-200a and miR-141 in melanoma pathway. Clin Cancer Drugs 2: 138-147, 2015.

43. Kim YK and Kim VN: Processing of intronic microRNAs. EMBO J 26: 775-783, 2007.

44. Lei H, Li H, Xie H, Du C, Xia Y and Tang W: Role of MiR-215 in Hirschsprung's Disease pathogenesis by targeting SIGLEC-8. Cell Physiol Biochem 40: 1646-1655, 2016.

45. Li N, Zhang QY, Zou JL, Li ZW, Tian TT, Dong B, Liu XJ, Ge S, Zhu Y, Gao J, et al: miR-215 promotes malignant progression of gastric cancer by targeting RUNX1. Oncotarget 7: 4817-4828, 2016.

46. Gao X, Cai Y and An R: miR 215 promotes epithelial to mesenchymal transition and proliferation by regulating LEFTY2 in endometrial cancer. Int J Mol Med 42: 1229-1236, 2018.

47. Wei Y, Sun J and Li X: MicroRNA-215 enhances invasion and migration by targeting retinoblastoma tumor suppressor gene 1 in high-grade glioma. Biotechnol Lett 39: 197-205, 2017.

This work is licensed under a Creative Commons Attribution-NonCommercial-NoDerivatives 4.0 International (CC BY-NC-ND 4.0) License. 